\title{
Soluble CD40 Ligand Levels in Otherwise Healthy Subjects With Impaired Fasting Glucose
}

\author{
Ilker Tasci, ${ }^{1}$ Teoman Dogru, ${ }^{1}$ Alper Sonmez, ${ }^{1}$ Halil Genc, ${ }^{1}$ Selim Kilic, ${ }^{2}$ Abdullah Olgun, ${ }^{3}$ Mahmut Gok, ${ }^{1}$ \\ Gokhan Erdem, ${ }^{1}$ and Selahattin Erikci ${ }^{1}$ \\ ${ }^{1}$ Department of Internal Medicine, Gulhane School of Medicine, 06018 Etlik, Ankara, Turkey \\ ${ }^{2}$ Department of Public Health, Gulhane School of Medicine, 06018 Etlik, Ankara, Turkey \\ ${ }^{3}$ Department of Biochemistry, Gulhane School of Medicine, 06018 Etlik, Ankara, Turkey
}

Received 1 June 2006; Accepted 7 August 2006

\begin{abstract}
Unlike diabetes mellitus and impaired glucose tolerance, it is not clear whether the subjects with impaired fasting glucose (IFG) are at increased risk of atherosclerosis and cardiovascular diseases. The CD40-CD40 ligand interaction is involved in the mechanism of atherosclerosis. We investigated whether soluble CD40L (sCD40L) as well as high sensitive C-reactive protein (hsCRP) levels are increased in subjects with IFG having no confounding factors for inflammation or atherosclerosis. Twenty four IFG subjects with no additional disorders and 40 appropriate healthy controls were studied. sCD40L and hsCRP levels in the IFG and control groups were similar. Blood pressures, total and LDL-cholesterol, and triglyceride levels were also similar, whereas HDL-cholesterol was lower and HOMA-IR indexes were higher in the IFG group. Though the sample size was small, the present data show that sCD40L seems not to alter in subjects with IFG suggesting that it might not be an independent risk factor for atherosclerosis.
\end{abstract}

Copyright (c) 2006 Ilker Tasci et al. This is an open access article distributed under the Creative Commons Attribution License, which permits unrestricted use, distribution, and reproduction in any medium, provided the original work is properly cited.

\section{INTRODUCTION}

Diabetes mellitus is a chronic progressive disease, which results in vascular complications due to macro- and microangiopathy [1]. Microangiopathy manifests itself as retinopathy, nephropathy, and neuropathy, whereas macroangiopathy is associated with accelerated atherosclerosis, which affects coronary, carotid, and peripheral arteries, and results in acute coronary syndrome, cerebrovascular disease, and peripheral vascular disease.

Impaired glucose tolerance (IGT) and impaired fasting glucose (IFG) are intermediate metabolic states between normal and diabetes mellitus [2]. Epidemiological data have shown that the risk of cardiovascular diseases (CVDs) increases linearly with increasing glycemia [3]. IGT has been linked to vascular complications by many authors, which was explained by the increase in 2-h glucose [4-6]. On the other hand, whether the subjects with IFG are at increased risk of vascular complications is not clear [7].

The CD40 ligand (CD40L), also known as CD154, is a transmembrane-bound protein, which is found not only on the cells of immunity but also on monocytes, macrophages, platelets, and endothelial cells [8]. It initiates a variety of inflammatory responses such as expression of cytokines, proteases, or leukocyte adhesion molecules upon interaction with CD40 receptor. Soluble and active forms of CD40 ligand (sCD40L) levels have been found to be increased in several conditions associated with atherosclerosis such as acute coronary syndrome [9], hypercholesterolemia [10], and IGT and diabetes mellitus [11].

Given the evidences that postprandial hyperglycemia and IGT are major risks for future CVDs [4-6], we designed the present study to investigate whether SCD40L as well as high sensitive C-reactive protein (hsCRP) levels are increased in individuals with IFG. In order to prevent any interference of confounding factors for inflammation, we studied a group having no additional disorders such as hypertension, diabetes mellitus, or obesity.

\section{PATIENTS AND METHODS}

\section{Participants}

Under the approval of the local ethics committee of Gulhane Medical School and by obtaining written informed consent, 24 subjects with isolated IFG and 40 age-, sex-, and BMImatched healthy controls were studied.

The inclusion criteria for the participants were no history of any metabolic or inflammatory disorders, absence 
of dyslipidemia [low-density lipoprotein (LDL)-cholesterol $<130 \mathrm{mg} / \mathrm{dl}$ ], hypertension (systolic blood pressure $<140$ $\mathrm{mmHg}$ and diastolic blood pressure $<90 \mathrm{mmHg}$ ), obesity [body mass index $(\mathrm{BMI})<30 \mathrm{~kg} / \mathrm{m}^{2}$ ], clinical evidence for either cardiovascular or peripheral artery diseases (history of acute myocardial infarction, coronary angioplasty, or bypass surgery, symptoms of angina, dyspnea, or claudicatio, and electrocardiographic abnormality) as well as rheumatic diseases, renal, hepatic, or thyroid dysfunction, concomitant medications (including herbal), regular alcohol, or drug consumption, smoking, and pregnancy. All subjects underwent a detailed physical examination and gave blood samples.

\section{Anthropometric measurements}

Weight and height were measured without shoes and in light clothing. BMI was calculated as weight divided by squared height $\left(\mathrm{kg} / \mathrm{m}^{2}\right)$. Systolic and diastolic blood pressures were measured using a manual mercury sphygmomanometer. Two readings were obtained five minutes apart in the sitting position, and the mean of the two was recorded as the blood pressure.

\section{Definition of glucose tolerance status}

All participants underwent standard oral glucose tolerance test (OGTT) according to the American Diabetes Association criteria [2]. A high-carbohydrate diet was applied three days before the test. At the baseline, blood was drawn under 12-hour fasting conditions in the morning. Then, $75 \mathrm{~g}$ glucose solution was given orally and a second venipuncture was performed 2 hours after the challenge. Normal glucose tolerance was defined as a fasting and 2-hour plasma glucose of $<100 \mathrm{mg} / \mathrm{dl}$ and $<140 \mathrm{mg} / \mathrm{dl}$, respectively. IFG was defined as fasting plasma glucose of 100-126 mg/dl and 2-hour plasma glucose of $<140 \mathrm{mg} / \mathrm{dl}$. Fasting plasma glucose of $100-126 \mathrm{mg} / \mathrm{dl}$ and 2-hour plasma glucose of 140-200 mg/dl were accepted as IGT. All the study and control subjects enrolled in the study had only isolated IFG or normal glucose tolerance.

\section{Biochemical parameters}

For biochemical analyses, all the serum samples were drawn between 08 : 00 and 08 : $30 \mathrm{AM}$ after a 12-hour fasting. The tubes were promptly centrifuged, and the plasma was separated and stored at $-70^{\circ} \mathrm{C}$. All plasma samples were run in the same assay. Glucose, total cholesterol, triglyceride (TG), and high-density lipoprotein (HDL)-cholesterol levels were measured by the enzymatic colorimetric method with Olympus AU 600 auto analyzer using reagents from Olympus Diagnostics, (GmbH, Hamburg, Germany). LDL-cholesterol was calculated by Fridewald's formula (12). The serum basal insulin was measured in duplicate by the coated tube method (DPC-USA).

Insulin resistance was calculated by homeostasis model assessment model (HOMA) using the formula HOMA-IR = Fasting insulin $(\mu \mathrm{U} / \mathrm{ml}) \times$ Fasting glucose $(\mathrm{mg} / \mathrm{dl}) / 405$ [12]
Low-IR values indicate high insulin sensitivity, whereas highHOMA values indicate low insulin sensitivity (insulin resistance).

\section{sCD40L and $h s C R P$}

Human sCD40L was measured by ELISA (Quantikine, R \& D Systems Inc, Minneapolis, USA) [sensitivity: (minimum detectable concentration $)=0.095 \mathrm{ng} / \mathrm{ml}$, intraCV: $4.0 \%$ and interCV: $6.8 \%$ ]. Serum hsCRP was determined by turbidimetric fixed rate method [13] by an automated analyzer (Olympus AU-2700, Mishima, Japan). Both tests were run in duplicate.

\section{Statistical analysis}

Results are reported as the mean \pm SD and median (for the skewed data). Differences between prediabetic and control groups were tested for significance by independent samples Student $t$ test and Mann-Whitney $U$ test. Differences were considered significant at $P<.05$. Because there has been no former data regarding sCD40L levels in such a study population, the sample size was not calculated by power analysis. The power of the study was calculated according to the results in the two groups.

\section{RESULTS}

The mean age, gender, BMI, blood pressure, fasting glucose, and plasma lipids of the participants are summarized in Table 1. Both groups had similar distribution of age, sex, and BMI. Systolic and diastolic blood pressures were also similar in the two groups.

Soluble CD40L levels in subjects with IFG and healthy controls were not different $(P=.14)$ (Figure 1). hsCRP levels were also comparable in the two groups $(P=.29)$. Total cholesterol, LDL-cholesterol, and TG levels were similar $(P=.40, P=.67$, and $P=.31$, resp $)$, whereas HDL cholesterol concentration was lower $(P<.001)$ in the IFG group compared to controls. HOMA indexes were higher in the IFG group as well $(P<.001)$.

The power of the study was calculated according to the results and found 0.57 for sCD40L, 0.71 for hsCRP, and 0.92 for HOMA.

\section{DISCUSSION}

In the present study, subjects with IFG had similar plasma sCD40L concentrations when compared to healthy individuals with normal glucose tolerance. hsCRP levels were not significantly different in the two groups as well. However, high levels of HOMA indexes indicated an insulin-resistant state in the IFG group.

Although IGT and IFG are not considered to be "diseases," they are associated with increased risk of development of overt diabetes mellitus [14], a true risk factor for morbidity, and mortality from CVDs [15]. However, the association between glucose status and the risk of CVDs starts below the 
TABLE 1: Comparison of characteristics and laboratory findings of the subjects with IFG and healthy controls. Data are expressed as mean value $\pm \mathrm{SD}$, median (min-max).

\begin{tabular}{l|ccc}
\hline & IFG $(n=24)$ & Controls $(n=40)$ & $P$ \\
\hline Age $(\mathrm{y})$ & $46.6 \pm 5.5$ & $47.4 \pm 7.5$ & $.64 \dagger$ \\
Gender (male/female) & $10 / 14$ & $17 / 23$ & $.13^{*}$ \\
Body mass index $\left(\mathrm{kg} / \mathrm{m}^{2}\right)$ & $28.3 \pm 3.3$ & $27.7 \pm 4.0$ & $.57 \dagger$ \\
Systolic blood pressure $(\mathrm{mmHg})$ & $125.4 \pm 8.6$ & $128.4 \pm 8.9$ & $.85 \dagger$ \\
Diastolic blood pressure $(\mathrm{mmHg})$ & $80.1 \pm 9.0$ & $80.2 \pm 5.4$ & $.44 \dagger$ \\
Total cholesterol $(\mathrm{mg} / \mathrm{dl})$ & $199.5 \pm 43.5$ & $208.6 \pm 36.7$ & $.40 \dagger$ \\
HDL-cholesterol $(\mathrm{mg} / \mathrm{dl})$ & $37.5(31-69)$ & $49(34-77)$ & $127(65-203)$ \\
LDL-cholesterol $(\mathrm{mg} / \mathrm{dl})$ & $129.5(79-221)$ & $118(50-397)$ & $.001 \neq$ \\
Triglyceride $(\mathrm{mg} / \mathrm{dl})$ & $137.5(65-447)$ & $77.5 \pm 8.3$ & $.37 \neq$ \\
Fasting glucose $(\mathrm{mg} / \mathrm{dl})$ & $110.7 \pm 8.1$ & $1.34(0.22-4.0)$ \\
hsCRP $(\mathrm{mg} / \mathrm{l})$ & $1.93(0.43-4.1)$ & $1.34(0.58-2.71)$ \\
HOMA-IR & $2.47(0.84-5.8)$ & $1.47(0.26-2.11)$ \\
sCD40L $(\mathrm{ng} / \mathrm{ml})$ & $1.0(0.04-3)$ & $.29 \neq$ \\
\hline
\end{tabular}

† Independent sample Student $t$ test, $\neq$ Mann-Whitney $U$ test, ${ }^{*}$ Chi-square test.

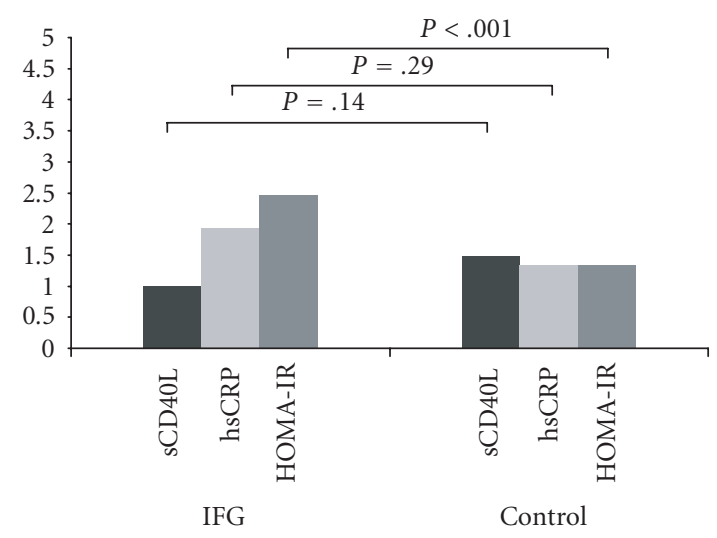

FIGURE 1: sCD40L (ng/mL) and hsCRP (mg/L) concentrations were similar, whereas HOMA-IR indexes were significantly higher in the IFG group in comparison with controls.

diagnostic threshold for diabetes mellitus [6]. Since 2-h glucose levels are known to increase the risk of vascular complications, IGT is considered to be a risk factor for CVDs [5]. However, the data related to the CVD risk in people with IFG are controversial. While several studies reported that even fasting hyperglycemia is associated with increased mortality (17-19), others concluded with less impressive results regarding an increased CVD risk in subjects with IFG $[7,16]$. The worse effects of postprandial hyperglycemia than fasting plasma glucose on atherosclerosis and related events have been defined by several mechanisms. Increased glycemia results in formation of intracellular reactive oxygen species [17] and advanced glycation end products (AGEs) from proteins and lipids in and outside the cells [18]. AGEs stimulation causes increased synthesis of reactive oxygen species in endothelial cells, proliferation of smooth muscle cells, decreased nitric oxide-mediated vasodilatation responses, as well as activation of mononuclear cells of immunity [18]. AGEs have also nonreceptor-mediated effects especially on extracellular matrix production in the vessel wall [19]. As was shown in people with IGT, hyperglycemia-induced oxidative stress triggers production of proinflammatory cytokines including hsCRP [20], which is an independent risk factor for future cardiovascular events [21]. Clinically, postchallenge hyperglycemia was reported to cause endothelial dysfunction together with increase in markers of oxidative stress [22], and related more strongly than fasting plasma glucose with carotid intima media thickness [23].

On the other hand, besides the evidence of harmful effects of postprandial hyperglycemia, the weak association between IFG and cardiovascular mortality in several studies might have been resultant from the unadjustment of clustering effects of other risk factors such as hypertension, dyslipidemia, obesity, or smoking. Determination of the global risk of IFG should include analyses of the other risk factors. Therefore, though the number of the study population is small, the present investigation addresses this question in a group of people with IFG having no confounders for atherosclerosis, and supports the results of previous studies $[7,16]$ concluding with no increase in the risk of CVDs in this category of glucose intolerance.

Endothelial dysfunction, usually induced by dyslipidemia, is one of the earliest events in the development of atherosclerosis, and inflammation is involved in the mechanism of atherosclerosis from the early phases [24]. Macrophages, upon activation by oxidized LDL, release a variety of substances such as cytokines and growth factors including tumor necrosis factor (TNF)- $\alpha$, interleukins (ILs), and sCD40L [25]. CD40L, which is not only expressed on macrophages but also synthesized by activated CD4 + cells, mast cells, basophils, eosinophils, and natural killer cells as well as endothelial cells, smooth muscle cells and platelets [8], is subsequently cleaved over a period of minutes to hours, generating a soluble fragment termed sCD40L, the biologically active form having similar proinflammatory effects on vascular cells [8]. Atherosclerosis and plaque formation is the result of chronic inflammation and 
wound-healing response, and CD40-CD40L system is a key regulator of communication between the cells of immunity in this process [26]. Engagement of CD40 with its ligand on the above-mentioned cells results in production of potent proinflammatory cytokines such as IL-1, IL-6, and IL-8 as well as increase in production of extracellular matrix and its proteases [26].

High plasma concentrations of sCD40L were proposed to be associated with increased vascular risk. Increased sCD40L was reported in patients with coronary heart disease [9], coronary restenosis after PTCA [27], and dyslipidemia [10]. Recently, it was also reported that, as the patients with diabetes mellitus, otherwise healthy people with IGT have increased plasma sCD40L levels when compared to controls [11]. In the present investigation, subjects with IFG who had no confounders for CVDs or inflammation did not have significant increase in sCD40L in their plasma. Given the evidences that postchallenge but not the fasting plasma glucose has stronger impacts on the risk of atherosclerosis and future cardiovascular events that are commonly accompanied by alterations in markers of chronic inflammation, we suggest that the people with IFG may not be under significant risk of CVDs unless their 2-hour glucose tolerance worsens. Besides sCD40L, the normal hsCRP levels in the IFG group further support this idea because hsCRP is a reliable marker for inflammation and is a predictor of future cardiovascular events [21].

In the present investigation, although their BMI, total cholesterol, triglyceride, sCD40L, and hsCRP concentrations were similar, the people with IFG were more insulin resistant than the controls. Subjects with IFG were reported to have tendency to be more insulin resistant than those with IGT $[28,29]$. Insulin resistance is a component of metabolic syndrome, a condition associated with atherosclerotic diseases [30]. However, current data related to an association of serum levels of inflammatory markers and structural indicators of atherosclerosis with insulin sensitivity is not clear. Insulin resistance calculated either by HOMA formula or intravenous glucose tolerance test was reported to correlate with CRP in people with IGT [31], indicating that the presence of resistance to biologic activity of insulin increases the risk of future cardiovascular events. On the other hand, it was also reported that insulin resistance correlates more strongly with hsCRP when present as part of insulin resistance syndrome [32], suggesting the common pathophysiological impacts of the determinants of the metabolic syndrome on atherosclerosis. However, several studies focusing on the carotid intima media thickness in nondiabetic subjects with [33] or without [34] glucose dysregulation concluded with no significant increase in structural findings of atherosclerosis in people with insulin resistance. Therefore, the interrelation between insulin resistance and serum markers of inflammation in people with IFG having no other components of metabolic syndrome or confounding factors for atherosclerosis should be further determined.

We found in our group of IFG that HDL cholesterol concentration was significantly lower compared to controls, whereas triglycerides as well as total and LDL cholesterol were similar. Igarashi et al have reported a decrease in HDL concentration as the glucose tolerance worsens in people with IGT [35]. Our results may indicate that decrease in HDL concentration starts early in the course of glucose intolerance. However, low HDL-cholesterol levels are not always accompanied by a predisposition to premature coronary heart disease [36]. Though an inverse correlation between HDL and CRP was previously described in patients with cardiovascular or atherosclerotic diseases [37], increased total and LDL cholesterol levels more strongly relate to atherosclerosis [38]. Nevertheless, more is needed to identify the prognostic importance of individual alterations in lipid profile and their association with inflammation markers in people with IFG.

Though the study population and the controls enrolled were selected specifically, the present study has two limitations. First, due to the narrow selection criteria the sample size was small to represent all people with IFG. Second, though it is simple, noninvasive, and known to be correlated well with clamp test [12], the HOMA formula used to calculate insulin sensitivity in this work is only an estimate and cannot be as accurate as the euglycemic hyperinsulinemic clamp method.

In conclusion, the results of the present study suggest no alterations in SCD40L as well as hsCRP levels in subjects with IFG, which contribute to the data that IFG alone might not be an independent risk factor for atherosclerosis. Studies with larger populations may provide further information regarding CD40-CD40L system and its interaction with other predictors of atherosclerosis in people with IFG.

\section{ACKNOWLEDGMENT}

This work was supported by the Gulhane School of Medicine Research Grant (AR-2005/2006).

\section{REFERENCES}

[1] Laight DW, Carrier MJ, Anggard EE. Endothelial cell dysfunction and the pathogenesis of diabetic macroangiopathy. Diabetes/Metabolism Research and Reviews. 1999;15(4):274-282.

[2] American Diabetes Association. Diagnosis and classification of diabetes mellitus. Diabetes Care. 2004;27(suppl 1):S5-S10.

[3] Shaw JE, Zimmet PZ, Hodge AM, et al. Impaired fasting glucose: how low should it go? Diabetes Care. 2000;23(1):34-39.

[4] Hanefeld M, Temelkova-Kurktschiev T. The postprandial state and the risk of atherosclerosis. Diabetic Medicine. 1997; 14(suppl 3):S6-S11.

[5] Saydah SH, Miret M, Sung J, Varas C, Gause D, Brancati FL. Postchallenge hyperglycemia and mortality in a national sample of U.S. adults. Diabetes Care. 2001;24(8):1397-1402.

[6] Haffner SM. Pre-diabetes, insulin resistance, inflammation and CVD risk. Diabetes Research and Clinical Practice. 2003; 61(suppl 1):S9-S18.

[7] Tominaga M, Eguchi H, Manaka H, Igarashi K, Kato T, Sekikawa A. Impaired glucose tolerance is a risk factor for cardiovascular disease, but not impaired fasting glucose: the Funagata Diabetes Study. Diabetes Care. 1999;22(6):920-924.

[8] Schönbeck U, Libby P. The CD40/CD154 receptor/ligand dyad. Cellular and Molecular Life Sciences. 2001;58(1):4-43. 
[9] Aukrust P, Müller F, Ueland T, et al. Enhanced levels of soluble and membrane-bound CD40 ligand in patients with unstable angina: possible reflection of T lymphocyte and platelet involvement in the pathogenesis of acute coronary syndromes. Circulation. 1999;100(6):614-620.

[10] Semb AG, Van Wissen S, Ueland T, et al. Raised serum levels of soluble CD40 ligand in patients with familial hypercholesterolemia: downregulatory effect of statin therapy. Journal of the American College of Cardiology. 2003;41(2):275-279.

[11] Gokulakrishnan K, Deepa R, Mohan V, Gross MD. Soluble Pselectin and CD40L levels in subjects with prediabetes, diabetes mellitus, and metabolic syndrome-the Chennai Urban Rural Epidemiology Study. Metabolism: Clinical and Experimental. 2006;55(2):237-242.

[12] Matthews DR, Hosker JP, Rudenski AS, Naylor BA, Treacher DF, Turner RC. Homeostasis model assessment: insulin resistance and $\beta$-cell function from fasting plasma glucose and insulin concentrations in man. Diabetologia. 1985;28(7):412419.

[13] Wasunna A, Whitelaw A, Gallimore R, Hawkins PN, Pepys MB. C-reactive protein and bacterial infection in preterm infants. European Journal of Pediatrics. 1990;149(6):424-427.

[14] Simpson RW, Shaw JE, Zimmet PZ. The prevention of type 2 diabetes-lifestyle change or pharmacotherapy? A challenge for the 21st century. Diabetes Research and Clinical Practice. 2003;59(3):165-180.

[15] Ashley EA, Raxwal V, Finlay M, Froelicher V. Diagnosing coronary artery disease in diabetic patients. Diabetes/Metabolism Research and Reviews. 2002;18(3):201-208.

[16] DECODE Study Group, the European Diabetes Epidemiology Group. Glucose tolerance and cardiovascular mortality. Comparison of fasting and 2-hour diagnostic criteria. Archives of Internal Medicine. 2001;161(3):397-405.

[17] Mohanty P, Hamouda W, Garg R, Aljada A, Ghanim H, Dandona P. Glucose challenge stimulates reactive oxygen species (ROS) generation by leucocytes. Journal of Clinical Endocrinology and Metabolism. 2000;85(8):2970-2973.

[18] Jenkins AJ, Best JD, Klein RL, Lyons TJ. Lipoproteins, glycoxidation and diabetic angiopathy. Diabetes/Metabolism Research and Reviews. 2004;20(5):349-368.

[19] Basta G, Schmidt AM, De Caterina R. Advanced glycation end products and vascular inflammation: implications for accelerated atherosclerosis in diabetes. Cardiovascular Research. 2004;63(4):582-592.

[20] Esposito K, Nappo F, Marfella R, et al. Inflammatory cytokine concentrations are acutely increased by hyperglycemia in humans: role of oxidative stress. Circulation. 2002;106(16):20672072.

[21] Mojiminiyi OA, Abdella N, Moussa MA, Akanji AO, Al Mohammedi $\mathrm{H}$, Zaki M. Association of C-reactive protein with coronary heart disease risk factors in patients with type 2 diabetes mellitus. Diabetes Research and Clinical Practice. 2002; 58(1):37-44.

[22] Ceriello A, Taboga C, Tonutti L, et al. Evidence for an independent and cumulative effect of postprandial hypertriglyceridemia and hyperglycemia on endothelial dysfunction and oxidative stress generation: effects of short- and long-term simvastatin treatment. Circulation. 2002;106(10):1211-1218.

[23] Hanefeld M, Koehler C, Henkel E, Fuecker K, Schaper F, Temelkova-Kurktschiev T. Post-challenge hyperglycaemia relates more strongly than fasting hyperglycaemia with carotid intima-media thickness: the RIAD study. Risk Factors in Impaired Glucose Tolerance for Atherosclerosis and Diabetes. Diabetic Medicine. 2000;17(12):835-840.
[24] Laight DW, Carrier MJ, Anggard EE. Endothelial cell dysfunction and the pathogenesis of diabetic macroangiopathy. Diabetes/Metabolism Research and Reviews. 1999;15(4):274-282.

[25] Szmitko PE, Wang C-H, Weisel RD, De Almeida JR, Anderson TJ, Verma S. New markers of inflammation and endothelial cell activation. Part I. Circulation. 2003;108(16):1917-1923.

[26] Phipps RP. Atherosclerosis: the emerging role of inflammation and the CD40-CD40 ligand system. Proceedings of the National Academy of Sciences of the United States of America. 2000;97(13):6930-6932.

[27] Cipollone F, Ferri C, Desideri G, et al. Preprocedural level of soluble CD40L is predictive of enhanced inflammatory response and restenosis after coronary angioplasty. Circulation. 2003;108(22):2776-2782.

[28] Nishi Y, Fukushima M, Suzuki H, et al. Insulin secretion and insulin sensitivity in Japanese subjects with impaired fasting glucose and isolated fasting hyperglycemia. Diabetes Research and Clinical Practice. 2005;70(1):46-52.

[29] Chen L-K, Lin M-H, Chen Z-J, Hwang S-J, Tsai S-T, Chiou ST. Metabolic characteristics and insulin resistance of impaired fasting glucose among the middle-aged and elderly Taiwanese. Diabetes Research and Clinical Practice. 2006;71(2):170-176.

[30] Cersosimo E, DeFronzo RA. Insulin resistance and endothelial dysfunction: the road map to cardiovascular diseases. Diabetes/Metabolism Research and Reviews. In press.

[31] Yudkin JS, Stehouwer CDA, Emeis JJ, Coppack SW. C-reactive protein in healthy subjects: associations with obesity, insulin resistance, and endothelial dysfunction: a potential role for cytokines originating from adipose tissue? Arteriosclerosis, Thrombosis, and Vascular Biology. 1999;19(4):972-978.

[32] Kang ES, Kim HJ, Ahn CW, et al. Relationship of serum high sensitivity C-reactive protein to metabolic syndrome and microvascular complications in type 2 diabetes. Diabetes Research and Clinical Practice. 2005;69(2):151-159.

[33] Snehalatha C, Vijay V, Suresh Mohan R, et al. Lack of association of insulin resistance and carotid intimal medial thickness in non-diabetic Asian Indian subjects. Diabetes/Metabolism Research and Reviews. 2001;17(6):444-447.

[34] Bonora E, Tessari R, Micciolo R, et al. Intimal-medial thickness of the carotid artery in nondiabetic and NIDDM patients: relationship with insulin resistance. Diabetes Care. 1997;20(4):627-631.

[35] Igarashi Y, Mita T, Mizuno R, et al. Characteristics of Japanese subjects who progress from normal to impaired glucose tolerance. Longitudinal study excluding changes in body weight. Endocrine Journal. 2005;52(6):683-689.

[36] Rader DJ, Ikewaki K, Duverger N, et al. Very low highdensity lipoproteins without coronary atherosclerosis. Lancet. 1993;342(8885):1455-1458.

[37] Tracy RP, Psaty BM, Macy E, et al. Lifetime smoking exposure affects the association of C-reactive protein with cardiovascular disease risk factors and subclinical disease in healthy elderly subjects. Arteriosclerosis, Thrombosis, and Vascular Biology. 1997;17(10):2167-2176.

[38] Greenland P, Knoll MD, Stamler J, et al. Major risk factors as antecedents of fatal and nonfatal coronary heart disease events. Journal of the American Medical Association. 2003;290(7):891-897. 


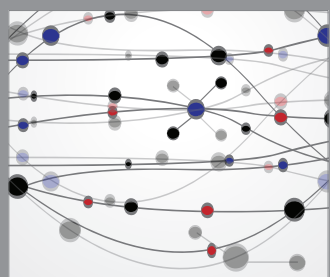

The Scientific World Journal
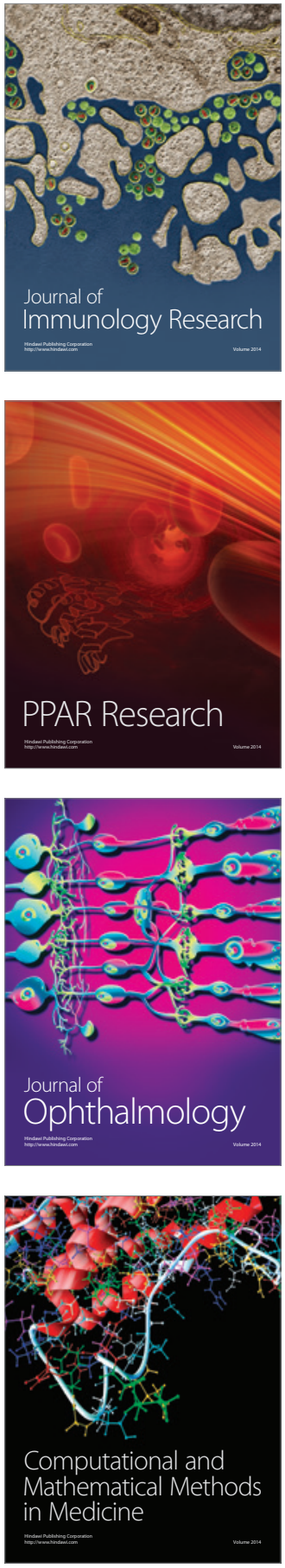

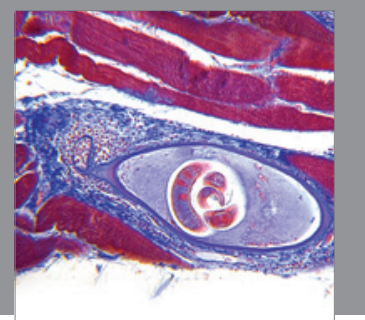

Gastroenterology

Research and Practice
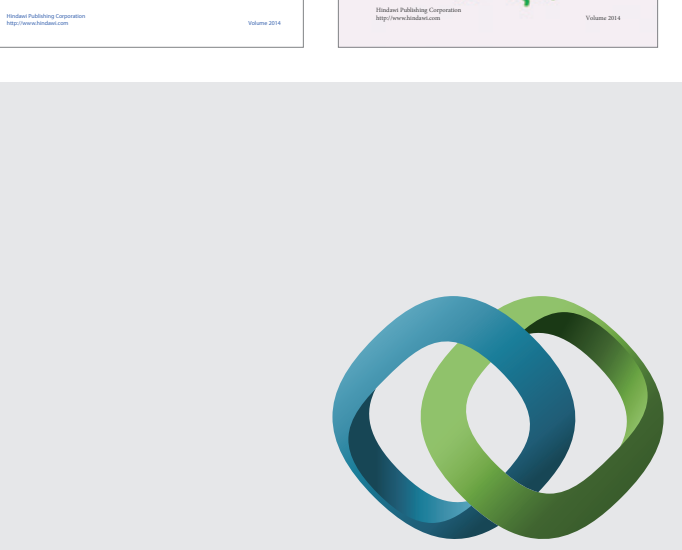

\section{Hindawi}

Submit your manuscripts at

http://www.hindawi.com
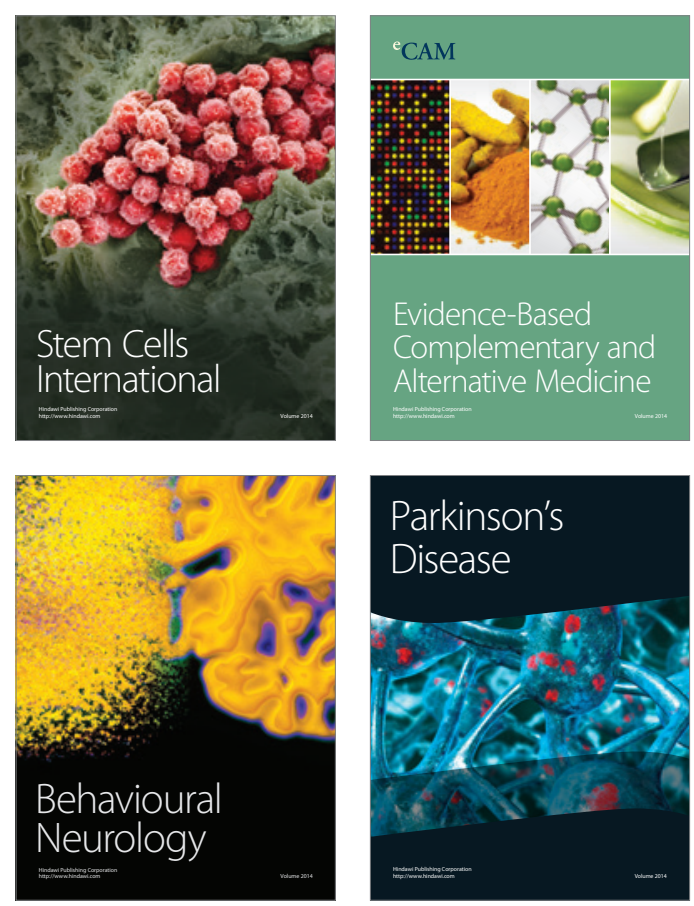

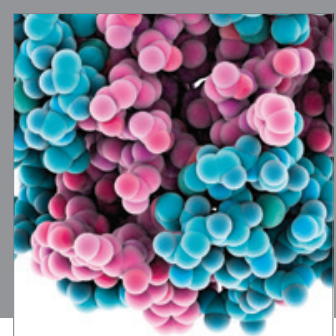

Journal of
Diabetes Research

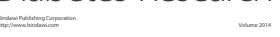

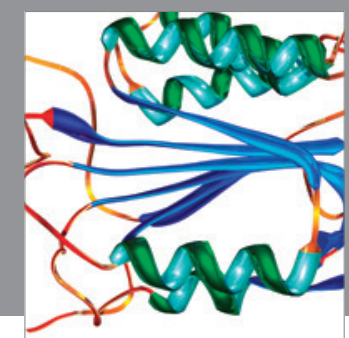

Disease Markers
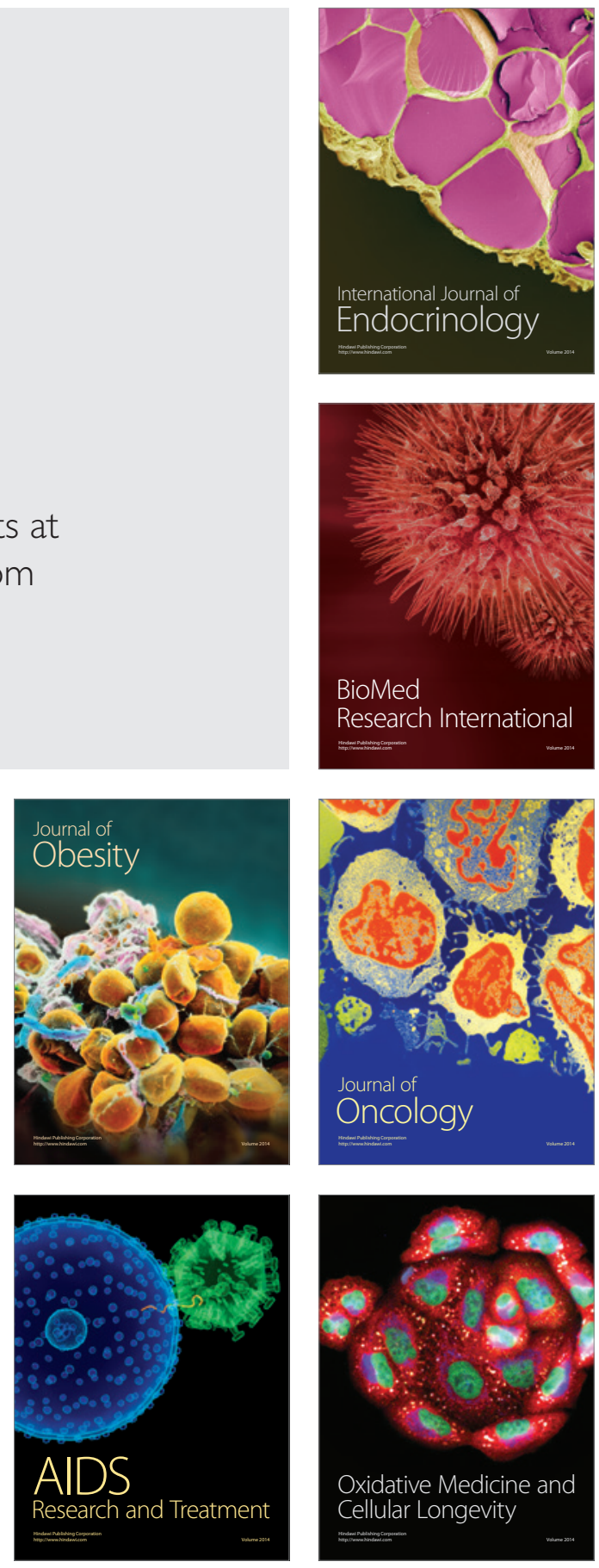